\title{
One-stage hybrid procedure for distal aortic arch disease: mid-term experience at a single center
}

\author{
Su-Wei Chen, Yong-Liang Zhong, Zhi-Yu Qiao, Cheng-Nan Li, Yi-Peng Ge, Rui-Dong Qi, Hai-Ou Hu, \\ Li-Zhong Sun, Jun-Ming Zhu \\ Department of Cardiovascular Surgery, Beijing Anzhen Hospital, Capital Medical University, Beijing Aortic Disease Center, Beijing Institute of \\ Heart, Lung and Blood Vessel Diseases, Beijing Engineering Research Center of Vascular Prostheses, Beijing, China \\ Contributions: (I) Conception and design: SW Chen, JM Zhu; (II) Administrative support: ZY Qiao, CN Li, YP Ge, LZ Sun; (III) Provision of \\ study materials or patients: SW Chen, YL Zhong; (IV) Collection and assembly of data: SW Chen, YL Zhong, ZY Qiao; (V) Data analysis and \\ interpretation: SW Chen, YL Zhong, HO Hu, RD Qi; (VI) Manuscript writing: All authors; (VII) Final approval of manuscript: All authors. \\ Correspondence to: Jun-Ming Zhu, MD. Department of Cardiovascular Surgery, Beijing Anzhen Hospital, 2 Anzhen Road, Beijing 100029, China. \\ Email: anzhenzjm@163.com.
}

Background: This study analyzes the outcomes of a one-stage hybrid procedure combining thoracic endovascular aortic repair (TEVAR) with extra-anatomic bypass in patients with distal aortic arch disease.

Methods: This retrospective study collected 103 hybrid procedures combining TEVAR with extraanatomic bypass (mean age, 62.2 \pm 9.3 years; 90 males) performed from January 2009 to January 2019 at Beijing Anzhen Hospital. We analyzed 30-day and mid-term outcomes including survival rate and the incidence of stroke, spinal cord injury (SCI), and endoleak.

Results: Five deaths (4.6\%) occurred within 30 days, including type I endoleak in Zone $1(\mathrm{n}=1)$, hemorrhagic shock $(n=1)$, stroke $(n=2)$, and stent migration $(n=1)$. Two patients developed SCI. The median follow-up time was 39.5 (interquartile range, 13.6-69.0) months. In all, 14 late deaths occurred; these were due to stroke $(n=2)$, severe pneumonia $(n=1)$, aortic rupture caused by type I endoleak $(n=3)$, and sudden death $(\mathrm{n}=8)$. Six late endoleaks occurred including three type I and one type II in Zone 1 and two type I in Zone 2. In a competing risks analysis, the incidences of reintervention at 7 years, late death, and survival without reintervention were $8 \%, 22 \%$, and $70 \%$, respectively. In a Cox risk model, stroke (HR, 21.602; $95 \%$ CI: 2.798-166.796; $\mathrm{P}=0.003)$ was the only risk factor for 30-day mortality. Stroke (HR, 19.484; $95 \%$ CI: 5.245-72.380; $\mathrm{P}<0.001)$, SCI (HR, 15.548; 95\% CI: 2.754-87.786; $\mathrm{P}=0.002)$, and endoleak (HR, 4.626; $95 \%$ CI: 1.068-20.040; $\mathrm{P}=0.041)$ were independent risk factors for long-term mortality.

Conclusions: The one-stage hybrid procedure provides acceptable mid-term results with good midterm patency of extra-anatomic bypass. Strict selection of patients suitable for hybrid repair can effectively improve the survival rate and reduce the incidence of complications. At the same time, close follow-up patients should receive close long-term follow-up after hybrid procedure.

Keywords: Hybrid; distal aortic arch; extra-anatomic bypass; thoracic endovascular aortic repair (TEVAR)

Submitted Jun 30, 2020. Accepted for publication Oct 25, 2020.

doi: $10.21037 /$ jtd-20-2338

View this article at: http://dx.doi.org/10.21037/jtd-20-2338

\section{Introduction}

Conventional open repair using moderate hypothermic circulatory arrest and selected antegrade cerebral perfusion is considered the primary strategy for aortic arch pathologies (1). Postoperative complications and mortality of this highly invasive procedure still seriously affect patient prognosis (2). Therefore, open repair may not be an optimal strategy for all patients, especially high-risk patients.

The development of endovascular repair technology 
has provided a new approach for the treatment of highrisk patients. Hybrid arch repair is a less invasive procedure that combines thoracic endovascular aortic repair (TEVAR) with extra-anatomic bypass. This procedure provides an alternative approach for patients who are unsuitable for conventional open repair. However, the effectiveness and safety of the hybrid procedure in the management of distal arch pathologies remain controversial. Hence, we review our mid-term experience in the management of distal arch pathologies using a one-stage hybrid procedure with extra-anatomic bypass. We present the following article in accordance with the STROBE reporting checklist (available at http://dx.doi.org/10.21037/jtd-20-2338).

\section{Methods}

The study was conducted in accordance with the Declaration of Helsinki (as revised in 2013). The study was approved by the Institutional Review Board of Beijing Anzhen Hospital of Capital Medical University (No. 2019073X), which waived the requirement for informed consent because of the retrospective nature of the analysis.

\section{Patients}

From January 2009 to January 2019, 112 consecutive patients were considered unsuitable for open repair and underwent a hybrid procedure combining retrograde TEVAR with extra-anatomic bypass at Beijing Anzhen Hospital. Three cases of two-stage hybrid procedure and six cases of zone 0 hybrid procedure were excluded. Finally, 103 patients were included in the present study. A multidisciplinary team determined whether a patient should undergo the hybrid procedure after a comprehensive assessment of the patient's condition. The multidisciplinary team included cardiovascular surgeons, anesthesiologists, perfusionists and intensive care specialists. The evaluation factors included age ( $>75$ years), frailty, aortic anatomy, the location and extent of the arch lesion, previous history of cardiac/aortic procedures, and severe comorbidities including severe impairment of cardiac, pulmonary, liver or renal function. All patients were diagnosed by computed tomographic angiography (CTA).

\section{Surgical technique}

All patients underwent the procedure under general anesthesia in a hybrid operating room.

\section{Debranching procedure}

Zone 1: the bilateral axillary arteries were dissociated through subclavian incisions, and the left common carotid artery (LCCA) was dissociated through the anterior edge of the sternocleidomastoid muscle. The extra-anatomic bypass from the right axillary artery (RAA) to left axillary artery (LAA) and the LCCA was established with a bifurcate "T" GORE-TEX ${ }^{\circledR}$ (W.L. Gore and Ass., Flagstaff, AZ, USA) prosthetic graft through a subcutaneous tunnel. The proximal end of the LCCA was ligated.

Zone 2: the bilateral axillary arteries were dissociated through subclavian incisions. The extra-anatomic bypass from the RAA to the LAA was established with a straight GORE-TEX ${ }^{\circledast}$ prosthetic graft through a subcutaneous tunnel (Figures 1,2).

\section{TEVAR procedure}

All patients underwent CTA at admission to evaluate the access route for TEVAR and measure the diameter of the aortic arch. A retrograde TEVAR procedure was performed via the femoral artery as previously described (3). Briefly, aortic angiography was performed first. The diameter of the aortic arch was re-measured using a calibrated pigtail catheter. The final diameter was determined by comparing the two measurements. Stent grafts were oversized by $10-15 \%$, and the minimum length of fixation along the inner curvature of the normal arch was at least $20 \mathrm{~mm}$. The strategy for aneurysm stent implantation was to completely cover the whole lesion. For aortic dissection, the strategy was to cover the entrance above the celiac artery and redirect the blood flow to the true lumen. Moreover, if the first stent was not a tapered stent, another tapered stent was deployed. The stent grafts overlapped with each other. The tapered stent was selected according to the taper rate of the true lumen. Finally, we embolized the proximal end of the left subclavian artery (LSA). For patients who developed spinal cord injury (SCI) after the procedure, cerebrospinal fluid (CSF) drainage was performed. Seven types of stent grafts were available: Zenith TX2 (Cook, Bloomington, IN, USA), Valiant (Medtronic, Santa Rosa, CA, USA), Grinkin (Grinkin Advanced Materials, Beijing, China), Hercules (MicroPort, Shanghai, China), Gore-TAG (W.L. Gore and Ass., Flagstaff, AZ, USA), Grimed (GRIMED, Beijing, China), and Ankura (Lifetech, Shenzhen, China). The selection of all devices was at the discretion of the surgeons. 

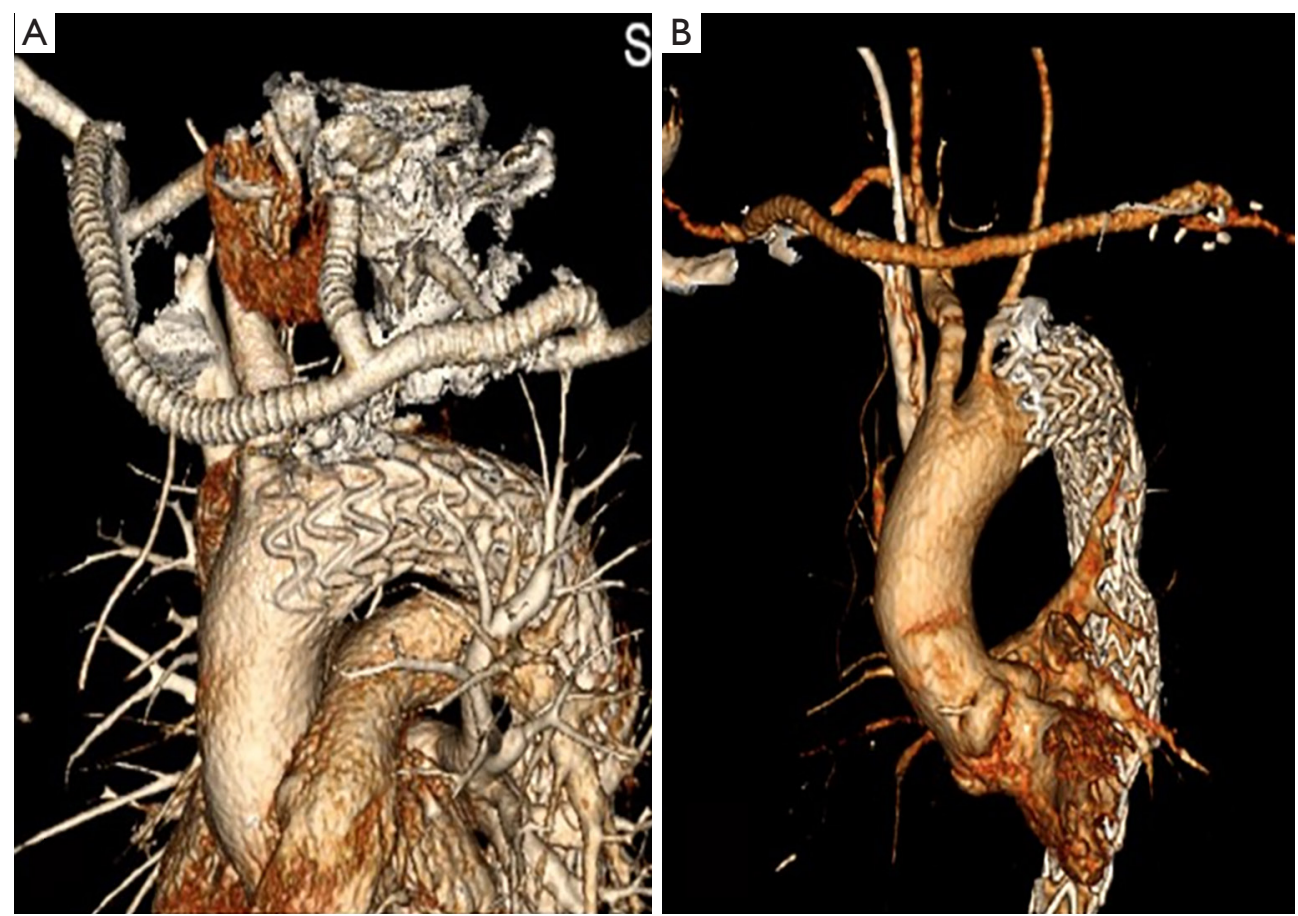

Figure 1 Hybrid procedures performed in different zones. (A) Hybrid procedure in Zone 1; (B) hybrid procedure in Zone 2.
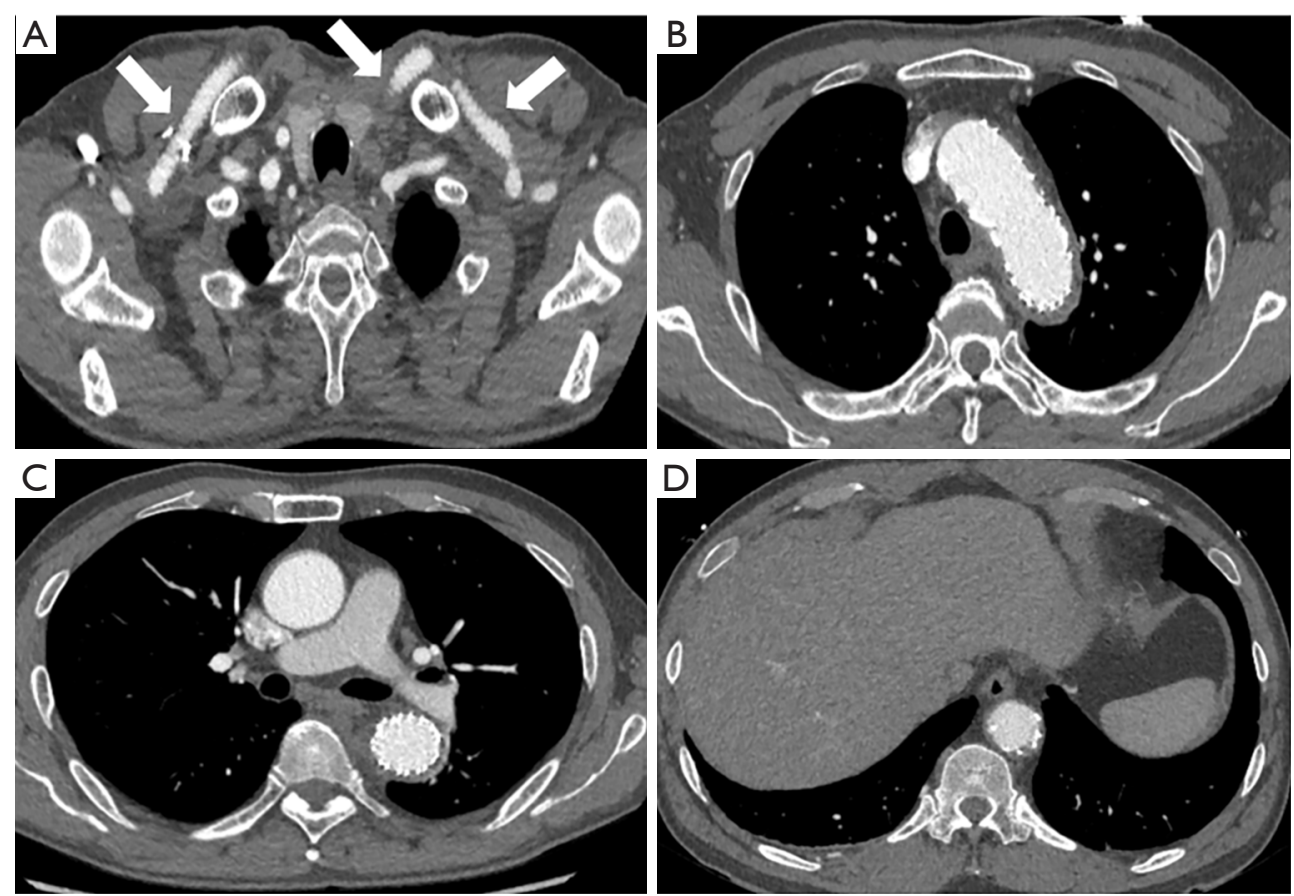

Figure 2 Postoperative CTA of the patient. (A) Good patency of extra-anatomic bypass; (B,C,D) the good continuity of the prosthetic graft without thrombus, endoleak or stent collapse. 
Table 1 Patient characteristics

\begin{tabular}{lc}
\hline Variables & Value $(\mathrm{n}=103, \%)$ \\
\hline Age & $62.2 \pm 9.3$ \\
Sex (male) & $90(87.4)$ \\
BMI $\left(\mathrm{kg} / \mathrm{m}^{2}\right)(\mathrm{IQR})$ & $25.8(23.8,27.7)$ \\
Smoking & $67(65.0)$ \\
COPD & $2(1.9)$ \\
Hypertension & $83(80.6)$ \\
CAD & $23(22.3)$ \\
Diabetes mellitus & $15(14.6)$ \\
Hyperlipemia & $6(5.8)$ \\
Renal insufficiency & $1(1.0)$ \\
Cerebrovascular disease & $12(11.7)$ \\
Previous cardiac/aortic surgery & $21(20.4)$ \\
LVEF (\%) (IQR) & $64.0(60.0,68.0)$ \\
ASA 3-4 & $97(94.2)$ \\
Elective & $72(69.9)$ \\
\hline
\end{tabular}

COPD, chronic obstructive pulmonary disease; CAD, coronary arterial disease; ASA, America society of anesthesiologist score; LVEF, left ventricular ejection fraction; IQR, interquartile range.

\section{Definition and follow-up}

The 30-day outcomes were the events that occurred within 30 days after the procedure and included mortality, stroke, SCI, and endoleak. Mid-term outcomes were events that occurred $>30$ days after the procedure and included the mid-term survival rate, stroke, SCI, and late endoleak. A previous cardiac/aortic procedure was defined as a history of cardiac/aortic opening or interventional therapy. Stroke was defined as any new global or focal neurologic deficit lasting $\geq 24$ hours with an acute lesion on brain imaging (4). SCI was defined as any new lower extremity deficit that was unrelated to an intracerebral event (4). Aortic event was defined as endoleak, aortic rupture, stent migration, distal stent graft-induced new entry, and aortic-related reintervention. The proximal landing zones of the aortic arch were defined following the Ishimaru classification (5). Follow-up was performed by telephone interview and hospital chart review. All patients were recommended to underwent CTA after discharge at 3, 6, 12 months, and then annually thereafter.

\section{Statistics}

Normally distributed continuous variables are expressed as the mean \pm standard deviation. Non-normally distributed continuous variables are presented as the median value and interquartile range (IQR). Categorical variables are expressed as numbers (percentages). The curves of overall survival and alive without aortic events were analyzed using the Kaplan-Meier method. Independent risk factors were analyzed by the multivariable Cox proportional risk model. The results are expressed as the hazard ratio (HR) and $95 \%$ confidence intervals (95\% CI). Competing risks of death and reintervention were analyzed via the Fine and Grey proportional hazards model. All statistics were 2 -sided tests, and $\mathrm{P}<0.05$ was considered statistically significant. All statistical data were analyzed using SPSS 25.0 (SPSS Inc., Chicago, USA) and GraphPad Prism for Windows 8.0 (GraphPad, La Jolla, CA, USA).

\section{Results}

\section{Patient characteristics}

The mean age was $62.2 \pm 9.3$ years. A total of 72 patients $(69.9 \%)$ underwent elective hybrid repair in this cohort. Twenty-one patients (20.4\%) had a previous cardiac/ aortic procedure, including 8 cases of TEVAR, 9 cases of percutaneous transluminal coronary intervention, 2 cases of the Bentall procedure, 1 case of descending aorta replacement, and 1 case of ascending aorta replacement. The 103 patients included 34 type $B$ aortic dissections (33.0\%), 32 aortic aneurysms (31.1\%), 19 pseudoaneurysms (18.4\%), 10 penetrating aortic ulcers (9.7\%), 2 intramural hematomas $(1.9 \%)$, and 6 penetrating aortic ulcers with intramural hematomas $(5.8 \%)$. The patient characteristics are shown in detail in Table 1.

\section{Surgical data}

All patients underwent hybrid procedures successfully, and no patient converted to open repair. Zones 1 and 2 hybrid procedures were performed in 54 cases $(52.4 \%)$ and 49 cases $(47.6 \%)$, respectively. The surgical time, length of stent grafts, proximal diameter of the stent grafts, ICU stay, mechanical ventilation duration, and hospitalization duration were $305.0(265.0,360.0) \mathrm{min}, 200.0(197.0,237.6) \mathrm{mm}$, $36.0(32.0,38.0) \mathrm{mm}, 19.0(16.0,21.0) \mathrm{h}, 10.0(6.0,17.0) \mathrm{h}$ 
Table 2 Surgical data

\begin{tabular}{lc}
\hline Variables & Value \\
\hline Hybrid procedure in different zones (\%) & \\
Zone 1 & $54(52.4)$ \\
Zone 2 & $49(47.6)$ \\
Hospitalization (d) (IQR) & $15.0(12.0,19.0)$ \\
Length of stent grafts (mm) (IQR) & $200.0(197.0,237.6)$ \\
Proximal diameter of stent grafts (mm) & $36.0(32.0,38.0)$ \\
IQR) & $19.0(16.0,21.0)$ \\
ICU stay (h) (IQR) & $305.0(265.0,360.0)$ \\
Surgical time (min) (IQR) & $10.0(6.0,17.0)$ \\
Mechanical ventilation (h) (IQR) &
\end{tabular}

$I Q R$, interquartile range.

and $15.0(12.0,19.0)$ days, respectively (Table 2$)$.

\section{0-day outcomes}

Five patients $(4.6 \%)$ died within 30 days. Two patients died of stroke. One patient was diagnosed with stent migration by CTA after the procedure; heart rate and blood pressure soon became difficult to maintain, and the patient eventually died. The other patient died of hemorrhagic shock, although no bypass anastomotic bleeding or any type of endoleak was found on various examinations. Another patient developed type I endoleak after the procedure and was treated with an emergency TEVAR procedure. Although the endoleak was blocked successfully, the vital signs of the patient could not be maintained, and the family members chose to give up treatment. He died the next day after leaving the hospital. Five more patients experienced postoperative complications. One patient had femoral artery thrombosis and underwent emergency thrombectomy. Another patient had poor incision healing of the femoral artery. Two patients developed SCI and underwent CSF drainage; one of the patients recovered well when discharged, while the other patient showed no significant improvement. The final patient had swelling of the left upper limb, but the related symptoms disappeared after 2 days (Table 3).

\section{Mid-term outcomes}

The median duration of follow-up time was 39.5 (13.6-
69.0) months. A total of 14 deaths occurred during followup, and these included stroke $(\mathrm{n}=2)$, severe pneumonia $(\mathrm{n}=1)$, aortic rupture caused by type I endoleak $(\mathrm{n}=3)$, and sudden death $(n=8)$. Late endoleak occurred in six individuals during the follow-up period, including five with type I and one with type II endoleak. Of the five patients with type I endoleak, one died while waiting for reintervention. One patient experienced a prosthetic graft infection followed by type I endoleak. But he refused reintervention and died due to aortic rupture. The other was diagnosed with type I endoleak in a local hospital but refused reintervention and died due to aortic rupture. Another patient also refused reintervention but was still alive at last follow-up. Only one patient with type I endoleak underwent a secondary TEVAR procedure and recovered well. One patient with type II endoleak received conservative treatment because the LSA was routinely embolized during the previous procedure and the return flow was small. Three more patients experienced late complications. One patient underwent secondary TEVAR because of distal stent graft-induced new entry. Late patency is satisfactory. During 39.5 months of followup, only one patient experienced slight stenosis of the extraanatomic bypass but no indication for reintervention. One patient had arm numbness, but there was no evidence of any nervous system injury or stenosis of the extra-anatomic bypass (Table 3).

The overall survival rate was $89.2 \%$ (95\% CI: $83.1-$ 95.3\%), 84.2\% (95\% CI: 76.8-91.6\%), 84.2\% (95\% CI: $76.8-91.6 \%)$ and $78.3 \%$ (95\% CI: $67.7-88.9 \%)$ at $1,3,5$ and 7 years, respectively (Figure $3 A$ ). The alive without aortic events rate was $92.7 \%$ (95\% CI: 87.4-98.0\%), 86.9\% (95\% CI: 79.5-94.3\%), 78.8\% (95\% CI: 68.6-89.0\%) and $63.6 \%$ (95\% CI: 48.9-78.3\%) at 1, 3, 5 and 7 years, respectively (Figure 3B). In the competing risks analysis, the incidence at 7 years was $8 \%$ for reintervention, $22 \%$ for late death and $70 \%$ for survival without reintervention (Figure 4). In the Cox proportional risk model, age was not an independent risk factor for either 30-day or late death. Stroke (HR, 21.602; 95\% CI: 2.798-166.796; P=0.003) was the only risk factor for 30-day mortality. Moreover, stroke (HR, 19.484; 95\% CI: 5.245-72.380; $\mathrm{P}<0.001$ ), SCI (HR, 15.548; 95\% CI: 2.754-87.786; $\mathrm{P}=0.002$ ), and endoleak (HR, 4.626; 95\% CI: 1.068-20.040; $\mathrm{P}=0.041$ ) were independent risk factors for long-term mortality, whereas cerebrovascular disease (HR, 1.071; 95\% CI: 0.218-5.266; $\mathrm{P}=0.932$ ) and a previous history of cardiac/aortic procedure (HR, 1.737; 95\% CI: 0.435-6.936; $\mathrm{P}=0.434$ ) were not (Table 4). 
Table 3 30-day and long-term outcomes

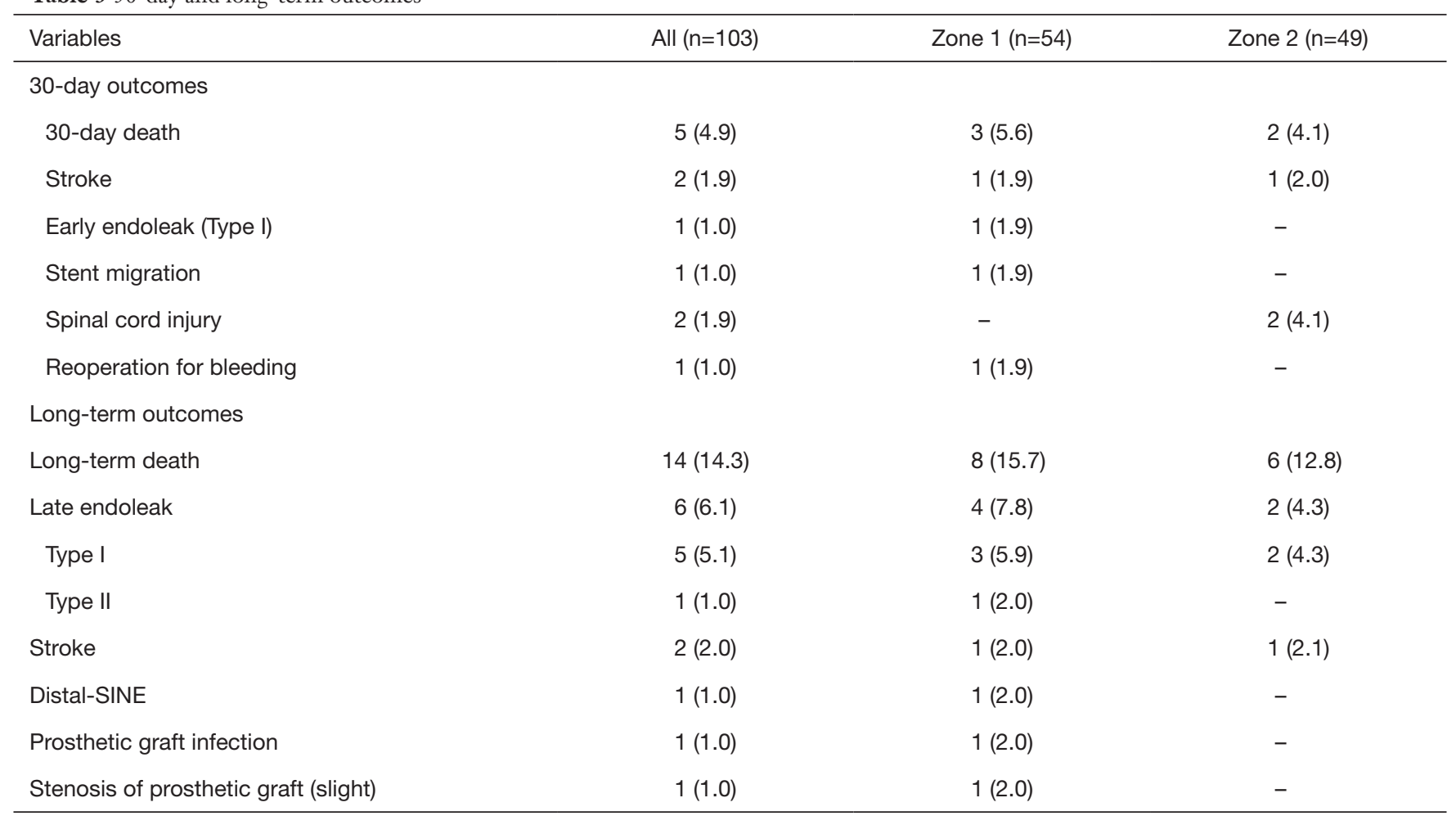

SINE, stent graft-induced new entry.

A

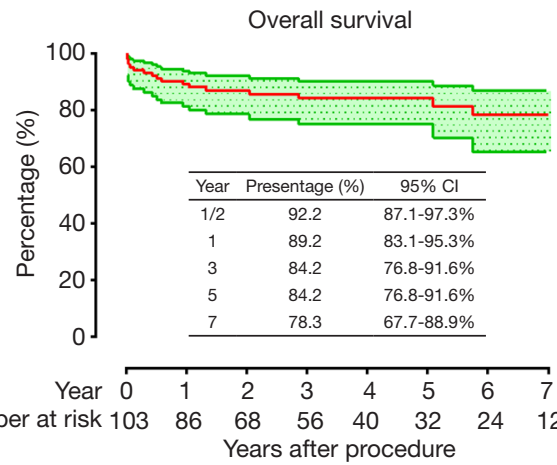

B

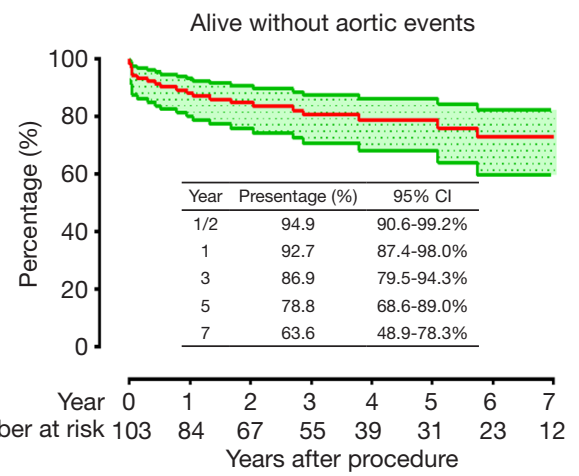

Figure 3 The overall survival of long-term follow-up (A); the alive without aortic events rate (B).

\section{Discussion}

Our results showed that the hybrid procedure with extraanatomic bypass achieved satisfactory 30 -day and midterm treatment outcomes for distal arch disease. The rate of overall survival was $78.3 \%$ at 7 years. Moreover, the incidences of postoperative complications were low in our cohort. According to our analysis, stroke is the only risk factor for 30-day death, while stroke, SCI and endoleak are independent risk factors for late death. Although our reintervention rate was $8 \%$, the results must be objectively understood. Reintervention was not performed in four patients with late endoleak. Of course, this outcome is related to the rapid progression of endoleak and the subjective decisions of patients and their families. In the present study, $63.6 \%$ of patients survived without aortic events. Therefore, strengthening the management of postoperative complications is important. 


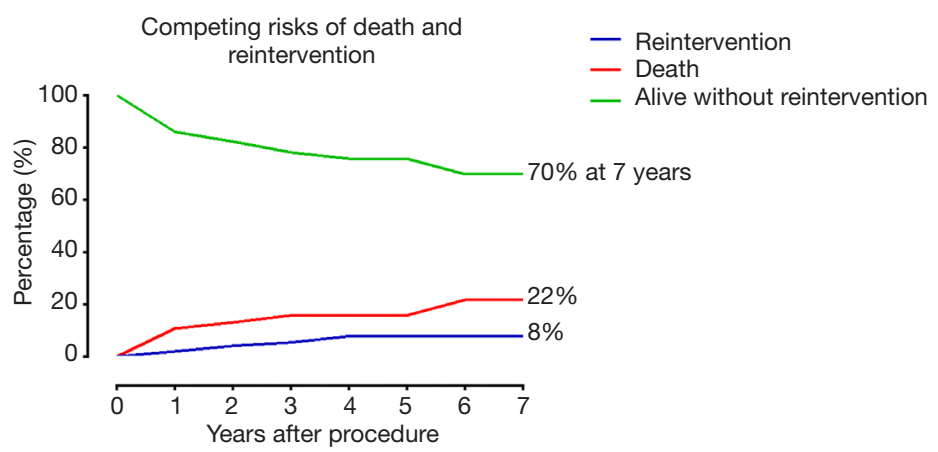

Figure 4 Competing risks of death and reintervention.

Table 4 Risk factors of mortality: Cox proportional hazard model

\begin{tabular}{lccc}
\hline Variables & HR & $95 \% \mathrm{Cl}$ & $\mathrm{P}$ value \\
\hline 30 -day mortality & & & $0.099-5.674$ \\
Age $\geq 65$ & 0.750 & $2.798-166.796$ & 0.781 \\
Stroke & 21.602 & & 0.003 \\
Long-term mortality & & $0.551-4.043$ & 0.432 \\
Age $\geq 65$ & 1.492 & $0.218-5.266$ & 0.932 \\
Cerebrovascular disease & 1.071 & $0.435-6.936$ & 0.434 \\
Previous cardiac/aortic procedure & 1.737 & $5.245-72.380$ & $<0.001$ \\
Stroke & 19.484 & $2.754-87.786$ & 0.002 \\
Spinal cord injury & 15.548 & $1.068-20.040$ & 0.041 \\
Endoleak & 4.626 & & \\
\hline
\end{tabular}

The satisfactory long-term results of supra-aortic branch bypass for treating occlusive disease led to its use to treat aortic diseases (6). However, two main problems were noted in the extra-anatomic bypass of supra-aortic branches. One was occlusion or stenosis, and the other was graft infection. Konstantinou et al. (7) reported that 15-month patency of extra-anatomic bypass was $99.1 \%$ in 201 patients. A retrospective study reported that overall extra-anatomical patency was $99 \%$ in 55 patients during 74.6 months of follow-up (8). In our cohort, late patency was 99\%. Only 1 patient $(1.0 \%)$ had slight stenosis of the extra-anatomic bypass without indication for reintervention. And 1 patient $(1.0 \%)$ developed prosthetic graft infection. With improvements in graft materials and aseptic techniques, these complications are bound to be rare. Based on the present study and the reported literature, the extraanatomic bypass approach is feasible. In addition, because of the possibility of asymptomatic occlusion of extra-anatomic bypass (6), all patients should receive close follow-up to avoid complications.

Compared with supra-aortic branch reconstruction, the TEVAR procedure was the more frequent cause of complications and death. Although we achieved acceptable results, the findings of other studies are inconsistent. De Rango et al. (9) reported a satisfactory long-term result with a five-year survival rate $70.9 \%$ of 104 patients. By contrast, Andersen et al. (10) reported that 13 patients (14.9\%) died within 30 days, and only $51 \%$ patients survived 5 years. A meta-analysis concluded that the hybrid procedure had high perioperative mortality ranging from $1.6 \%$ to $25.0 \%$ (11). Moreover, the high rates of postoperative complications and reintervention seriously affect patient prognosis. According to the literature, $42 \%$ of patients develop late complications (12), and $32 \%$ of patients undergo reintervention during the follow-up (13). New lesions in degenerative aortic disease 
inevitably increase the likelihood of intervention. Although elective open conversion for reintervention is acceptable in treating late complications (12), open reintervention increases the risk of death for these high-risk patients due to the complexity of complications. Therefore, the choice of reintervention methods should be made based on the complexity of complications and the patient's health status.

Endoleak was the most common complication, with an incidence of $0-42 \%$ (14-16). Untreated endoleak increases the likelihood of death (17). The predictors of endoleak include anatomic abnormalities, angulation of the arch, the diameter of the aneurysmal aorta, the lengths of the stent grafts covering the aorta, the number of stent grafts, the length of the proximal landing zone, and zone types $(13,18-20)$. The incidence of endoleak is higher in zone 1 hybrid repair, similar to our results (20). The total debranching procedure seems to prevent endoleak (14), but zone 0 repair increases the risk of death (21). Moreover, stent grafts have a shortening characteristic. If the proximal landing zone is closer to zone 0 , the length of the stent grafts that actually provides coverage will be less than its original length. In order to avoid endoleak, stent grafts should maintain sufficient proximal and distal landing zones so that endografts are placed on the normal aorta. Furthermore, the strategy of stent implantation should be based on individual pathology and prognosis.

Stroke is a serious postoperative complication with a high incidence ranging from $0 \%$ to $28.6 \%(11,22)$. Embolism, hemodynamic hypoperfusion, rigid wires, and stiff delivery systems play important roles in stroke $(23,24)$. The anterograde approach may be safer than the retrograde approach (25). However, the anterograde TEVAR procedure still has several limitations associated with dissection, aortic angulations and tortuosity, and requires assistance by cardiopulmonary bypass. Moreover, transapical endovascular repair has developed in recent years (26). Transapical endovascular repair for TEVAR provides a new approach for patients who are unsuitable for routine approaches, but long-term evidence from large cohort studies is limited. In addition, objective evidence demonstrating the advantages and disadvantages of these approaches is lacking.

The incidence of SCI is relatively low and ranges from $2.2 \%$ to $3.6 \%(27,28)$. Hemodynamic stability and proper postoperative blood pressure control reduce the risk of SCI $(29,30)$. The two-stage hybrid procedure is also considered an effective method to prevent SCI, but the optimal interval time between the two stages has not been clearly established $(13,31)$. A prolonged interval time for the second-stage procedure increases the risk of death. Therefore, a onestage hybrid procedure should be performed in patients who cannot tolerate a two-stage procedure (32). Moreover, our results showed that with regard to maintaining stable haemodynamics and stabilizing perioperative blood pressure, the one-stage hybrid procedure increased neither the risk of aortic rupture nor the incidence of SCI.

Hybrid arch repair is considered an alternative treatment for high-risk patients. Currently, multidisciplinary consultation, American Society of Anesthesiologists scores, or the European system for cardiac operative risk evaluation II (EuroSCORE II) are the main ways to identify high-risk patients (33-35). However, no study has demonstrated that the aforementioned methods are effective in hybrid repair. In addition, EuroSCORE II does not accurately estimate the surgical risk of patients $(10,34)$ because it was originally developed to predict the mortality of cardiac surgery rather than that of aortic surgery. Moreover, hybrid repair is slightly different from traditional open repair. Hybrid repair has its own characteristics, such as the application of endovascular stents and differences in baseline characteristics and types of postoperative complications. Although further studies are needed to confirm our opinion, we suggest that the current scoring system may not be a good evaluator of hybrid repair.

\section{Limitations}

Our study has some limitations. First, it was a singlecenter retrospective study. Due to its nonrandomized and retrospective nature, the results are susceptible to selective bias. Second, since hybrid procedures are not the primary treatment for all patients at our center, the sample size was limited. Third, this cohort consisted of patients with different arch pathologies, which may also have affected the outcomes.

\section{Conclusions}

Our study reveals that the one-stage hybrid procedure provides acceptable mid-term results with good late patency of extra-anatomic bypass. Strict selection of patients suitable for hybrid repair can effectively improve the survival rate and reduce the incidence of complications. In addition, patients should receive close long-term follow-up after hybrid procedure. 


\section{Acknowledgments}

Funding: This work was supported by the Natural Science Foundation of China (No. 81970393) and the Beijing Major Science and Technology Projects from the Beijing Municipal Science and Technology Commission (No. Z191100006619093).

\section{Footnote}

Reporting Checklist: The authors have completed the STROBE reporting checklist. Available at http://dx.doi. org/10.21037/jtd-20-2338

Data Sharing Statement: Available at http://dx.doi. org/10.21037/jtd-20-2338

Conflicts of Interest: All authors have completed the ICMJE uniform disclosure form (available at http://dx.doi. org/10.21037/jtd-20-2338). The authors have no conflicts of interest to declare.

Ethical Statement: The authors are accountable for all aspects of the work in ensuring that questions related to the accuracy or integrity of any part of the work are appropriately investigated and resolved. The study was conducted in accordance with the Declaration of Helsinki (as revised in 2013). The study was approved by the Institutional Review Board of Beijing Anzhen Hospital of Capital Medical University (No. 2019073X), which waived the requirement for informed consent because of the retrospective nature of the analysis.

Open Access Statement: This is an Open Access article distributed in accordance with the Creative Commons Attribution-NonCommercial-NoDerivs 4.0 International License (CC BY-NC-ND 4.0), which permits the noncommercial replication and distribution of the article with the strict proviso that no changes or edits are made and the original work is properly cited (including links to both the formal publication through the relevant DOI and the license). See: https://creativecommons.org/licenses/by-nc-nd/4.0/.

\section{References}

1. Ma WG, Zhu JM, Zhang W, et al. Frozen elephant trunk with total arch replacement for type A aortic dissections: Does acuity affect operative mortality? J Thorac
Cardiovasc Surg 2014;148:963-70; discussion 970-2.

2. Furutachi A, Takamatsu M, Nogami E, et al. Early and mid-term outcomes of total arch replacement with the frozen elephant trunk technique for type A acute aortic dissection. Interact Cardiovasc Thorac Surg 2019;29:753-60.

3. Xu SD, Huang FJ, Yang JF, et al. Early and midterm results of thoracic endovascular aortic repair of chronic type B aortic dissection. J Thorac Cardiovasc Surg 2010;139:1548-53.

4. Lombardi JV, Hughes GC, Appoo JJ, et al. Society for Vascular Surgery (SVS) and Society of Thoracic Surgeons (STS) Reporting Standards for Type B Aortic Dissections. Ann Thorac Surg 2020;109:959-81.

5. Fillinger MF, Greenberg RK, McKinsey JF, et al. Reporting standards for thoracic endovascular aortic repair (TEVAR). J Vasc Surg 2010;52:1022-33, 33.e15.

6. Ozsvath KJ, Roddy SP, Darling RC, 3rd, et al. Carotidcarotid crossover bypass: is it a durable procedure? J Vasc Surg 2003;37:582-5.

7. Konstantinou N, Debus ES, Vermeulen CFW, et al. Cervical Debranching in the Endovascular Era: A Single Centre Experience. Eur J Vasc Endovasc Surg 2019;58:34-40.

8. Martin G, Riga C, Gibbs R, et al. Short- and Long-term Results of Hybrid Arch and Proximal Descending Thoracic Aortic Repair: A Benchmark for New Technologies. J Endovasc Ther 2016;23:783-90.

9. De Rango P, Cao P, Ferrer C, et al. Aortic arch debranching and thoracic endovascular repair. J Vasc Surg 2014;59:107-14.

10. Andersen ND, Williams JB, Hanna JM, et al. Results with an algorithmic approach to hybrid repair of the aortic arch. J Vasc Surg 2013;57:655-67; discussion 666-7.

11. Cao P, De Rango P, Czerny M, et al. Systematic review of clinical outcomes in hybrid procedures for aortic arch dissections and other arch diseases. J Thorac Cardiovasc Surg 2012;144:1286-300, 300.e1-2.

12. Joo HC, Youn YN, Kwon JH, et al. Late complications after hybrid aortic arch repair. J Vasc Surg 2019;70:1023-30.e1.

13. Gurupatham S, Qadura M, Andrinopoulos T, et al. Single Institution Experience with Hybrid Endovascular and Surgical Repairs Involving the Distal Aortic Arch. Ann Vasc Surg 2020;62:223-31.

14. Gelpi G, Vanelli P, Mangini A, et al. Hybrid aortic arch repair procedure: reinforcement of the aorta for a safe and durable landing zone. Eur J Vasc Endovasc Surg 
2010;40:709-14.

15. Bavaria J, Vallabhajosyula P, Moeller P, et al. Hybrid approaches in the treatment of aortic arch aneurysms: postoperative and midterm outcomes. J Thorac Cardiovasc Surg 2013;145:S85-90.

16. Antoniou GA, Mireskandari M, Bicknell CD, et al. Hybrid repair of the aortic arch in patients with extensive aortic disease. Eur J Vasc Endovasc Surg 2010;40:715-21.

17. Caronno R, Piffaretti G, Tozzi M, et al. Emergency endovascular stent-graft treatment for acute thoracic aortic syndromes. Surgery 2006;140:58-65.

18. Piffaretti G, Mariscalco G, Lomazzi C, et al. Predictive factors for endoleaks after thoracic aortic aneurysm endograft repair. J Thorac Cardiovasc Surg 2009;138:880-5.

19. Czerny M, Grimm M, Zimpfer D, et al. Results after endovascular stent graft placement in atherosclerotic aneurysms involving the descending aorta. Ann Thorac Surg 2007;83:450-5.

20. Kotelis D, Geisbusch P, Attigah N, et al. Total vs hemiaortic arch transposition for hybrid aortic arch repair. J Vasc Surg 2011;54:1182-6.e2.

21. Geisbusch P, Kotelis D, Muller-Eschner M, et al. Complications after aortic arch hybrid repair. J Vasc Surg 2011;53:935-41.

22. Hiraoka A, Chikazawa G, Tamura K, et al. Clinical outcomes of different approaches to aortic arch disease. J Vasc Surg 2015;61:88-95.

23. Preventza O, Garcia A, Cooley DA, et al. Total aortic arch replacement: A comparative study of zone 0 hybrid arch exclusion versus traditional open repair. J Thorac Cardiovasc Surg 2015;150:1591-8; discussion 1598-600.

24. Hiraoka A, Chikazawa G, Totsugawa T, et al. Objective analysis of midterm outcomes of conventional and hybrid aortic arch repair by propensity-score matching. J Thorac Cardiovasc Surg 2017;154:100-6.e1.

Cite this article as: Chen SW, Zhong YL, Qiao ZY, Li CN, Ge YP, Qi RD, Hu HO, Sun LZ, Zhu JM. One-stage hybrid procedure for distal aortic arch disease: mid-term experience at a single center. J Thorac Dis 2020;12(12):7117-7126. doi: $10.21037 /$ jtd-20-2338
25. Roselli EE, Soltesz EG, Mastracci T, et al. Antegrade delivery of stent grafts to treat complex thoracic aortic disease. Ann Thorac Surg 2010;90:539-46.

26. Murakami T, Nishimura S, Hosono M, et al. Transapical Endovascular Repair of Thoracic Aortic Pathology. Ann Vasc Surg 2017;43:56-64.

27. Bunger CM, Kische S, Liebold A, et al. Hybrid aortic arch repair for complicated type B aortic dissection. J Vasc Surg 2013;58:1490-6.

28. Moulakakis KG, Mylonas SN, Markatis F, et al. A systematic review and meta-analysis of hybrid aortic arch replacement. Ann Cardiothorac Surg 2013;2:247-60.

29. Oishi Y, Sonoda H, Ushijima T, et al. Single-stage hybrid total arch replacement for extended arch aneurysms. J Vasc Surg 2019;69:1719-25.

30. Ranney DN, Cox ML, Yerokun BA, et al. Long-term results of endovascular repair for descending thoracic aortic aneurysms. J Vasc Surg 2018;67:363-8.

31. Yoshitake A, Iida Y, Yamazaki M, et al. Midterm Results of 2-Stage Hybrid Arch Repair for Extensive Aortic Arch Aneurysms. Ann Vasc Surg 2019;56:97-102.

32. Bibiloni Lage I, Calsina Juscafresa L, Delgado Dominguez C, et al. Hybrid Repair of Aortic Arch Aneurysms with Endografting of the Ascending Aorta. J Card Surg 2016;31:341-7.

33. Faure EM, Canaud L, Marty-Ane C, et al. Hybrid aortic arch repair for dissecting aneurysm. J Thorac Cardiovasc Surg 2016;152:162-8.

34. Cazavet A, Alacoque X, Marcheix B, et al. Aortic arch aneurysm: short- and mid-term results comparing open arch surgery and the hybrid proceduredagger. Eur J Cardiothorac Surg 2016;49:134-40.

35. Canaud L, Joyeux F, Ziza V, et al. Hemi-aortic arch debranching for hybrid aortic arch repair by sequential transposition of the left common carotid and subclavian arteries. J Thorac Cardiovasc Surg 2013;145:764-7. 